\title{
Design and comparison of the Cs ovens for the test facilities ELISE and SPIDER
}

\author{
Sofia Cristofaro ${ }^{1, a}$, Markus Fröschle ${ }^{1}$, Alessandro Mimo ${ }^{1}$, Andrea Rizzolo $^{2}$, Michela De Muri ${ }^{2}$, \\ Marco Barbisan ${ }^{2}$ and Ursel Fantz ${ }^{1}$ \\ ${ }^{1}$ Max-Planck-Institut für Plasmaphysik, Boltzmannstr. 2, 85748 Garching, Germany \\ ${ }^{2}$ Consorzio RFX, Corso Stati Uniti 4, 35127 Padova, Italy \\ ${ }^{a}$ Corresponding author: sofia.cristofaro@ipp.mpg.de
}

\begin{abstract}
Negative ion sources for fusion rely on the formation of negative hydrogen (or deuterium) ions by conversion of atomic hydrogen and positive hydrogen ions at a low work function caesiated surface. Cs is thus evaporated into the source to decrease the surface work function, which may change due to the removal and redistribution of Cs during plasma phases. To maintain a temporarily stable low work function during one hour plasma, continuous evaporation of caesium is required, and this is performed by temperature controlled Cs ovens. The Cs ovens for ELISE (IPP Garching) and SPIDER (Consorzio RFX) are based on the evaporation of liquid Cs from a reservoir located at one end of the oven, and the evaporation of Cs is controlled by the reservoir temperature. The ampoule Cs oven of ELISE is in operation since 2015, allowing for controllable and stable evaporation. The SPIDER oven is based on the ELISE oven, though it required significant changes due to the vacuum environment and the oven location (at the back-plate instead of the sidewalls), leading to a different design of the oven and the nozzle. First investigations on the SPIDER oven in a dedicated test stand show that Cs evaporation is controllable, stable and reproducible.
\end{abstract}

\section{Introduction}

Negative ion sources for the neutral beam injection system of the fusion experiment ITER (Cadarache, France) must deliver high energy negative hydrogen (or deuterium) ion beams accelerated up to $1 \mathrm{MeV}$ with an extracted current density of $329 \mathrm{Am}^{-2}\left(286 \mathrm{Am}^{-2}\right.$ for deuterium) over an ion source surface of $1 \times 2 \mathrm{~m}^{2}$ (with an extraction area of $0.2 \mathrm{~m}^{2}$ ) and with pulse lengths of up to one hour [1]. Electrons are inevitably co-extracted, and the ratio between the co-extracted electron to extracted negative ion current must be below one. The ion sources must operate at a gas pressure of $0.3 \mathrm{~Pa}$, and the negative ions are mainly formed by conversion of atomic hydrogen and positive hydrogen ions from the plasma impinging onto a low work function surface [2]. In order to lower the work function of the converter surface, Cs is evaporated in the source [3] since it has the lowest work function among the elements $(2.1 \mathrm{eV}$ for bulk Cs [4]). Consequently, Cs is deposited on the source surfaces, and the interaction between the plasma and the deposited Cs leads to a removal and redistribution of Cs from the surfaces [5], leading also to a degradation of the work function of the converter surface during long and continuous plasma operation [6]. Since electrons are the minority species, a rise of the work function would then lead to an increase of the co-extracted electron current. Thus, it is necessary to sustain a sufficient Cs flux towards the plasma grid in order to maintain a temporally stable work function. However, there is an operative upper limit on the Cs content in the source, given by the fact that $\mathrm{Cs}$ changes the RF coupling and causes breakdowns in the accelerator downstream of the source [7]. At the ion sources Cs is evaporated by means of temperature controlled Cs ovens which must ensure adjustable, continuous and stable evaporation [2].
The test facility ELISE at Max-Planck-Institut für Plasmaphysik (Garching, Germany) is equipped with a source half of the size of the ITER ion source $\left(1 \times 1 \mathrm{~m}^{2}\right)$ and is in operation since 2013 [8]. Two liquid Cs ovens are installed at the sidewalls. At present, the ITER requirements have not yet been fulfilled at ELISE for the entire length of the plasma pulse in deuterium [9] because of the continuous increase of the co-extracted electron current, probably due to a not temporally stable work function of the converter surface.

The test facility SPIDER at Consorzio RFX (Padova, Italy) is equipped with a full ITER-size ion source [10] and has started first operation in 2018. Cs operation is foreseen in future, and three Cs ovens will be installed at the back-plate of the ion source. Contrarily to ELISE, the SPIDER source is fully in vacuum (as for ITER), including the ovens, hence a new oven design was required. The ovens have been recently manufactured and first tests have been performed at the dedicated laboratory experiment CATS (CAesium Test Stand) at Consorzio RFX [11].

\section{Cs oven at ELISE}

The ampoule Cs oven for ELISE was designed and manufactured at IPP Garching, and it is routinely applied since 2015. A CAD drawing of the Cs oven is shown in Figure 1a. The oven is mainly made of staineless steel tubes and off-the-shelf tube fittings, while the injection tube is made of nickel plated copper: $\mathrm{Cu}$ ensures a good heat conductivity, while $\mathrm{Ni}$ is chemically inert to Cs. A sealed glass ampoule containing $1 \mathrm{~g}$ of pure Cs is located almost vertically in a stainless steel tube. The ampoule can be easily cracked from the outside by pressing the tube. Once the ampoule is broken, the liquid Cs is collected in the reservoir, which is in contact with a heating sleeve and 

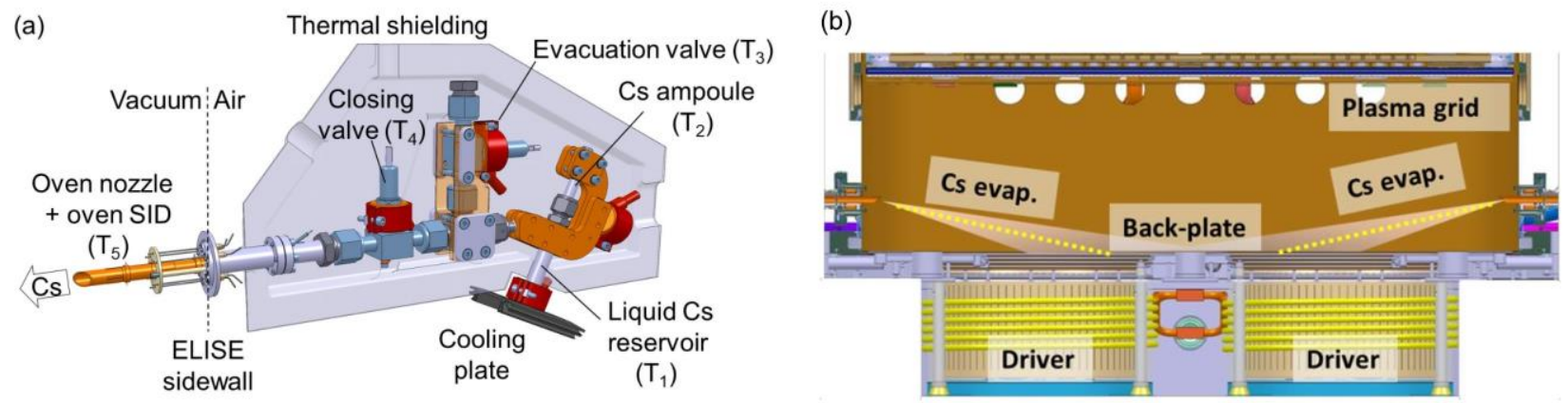

Fig. 1. (a) CAD drawing of the Cs ampoule oven operated at ELISE. The main components are highlighted and the positions of the five thermocouples are indicated by $T_{i}$, with $i=1, . ., 5$. (b) Top view of ELISE ion source with position and orientation of the two Cs oven nozzles.

a cooling plate and is the coldest spot of the oven. Cs is evaporated by heating the reservoir, whose temperature determines the evaporation rate and is typically between $80^{\circ} \mathrm{C}$ and $130^{\circ} \mathrm{C}$. The rest of the oven is kept at higher temperature (around $280^{\circ} \mathrm{C}$ ) to avoid the presence of cold spots (where Cs could deposit) and to ensure high Cs transport to the experiment vessel. A thermal shielding made of ceramic fiber in a silicon case insulates the oven body, while the cooling plate is located outside the thermal shielding to maintain the lower temperature of the reservoir, and the free air convection allows decreasing rapidly the reservoir temperature when needed. The oven temperature is feedback controlled by five heating sleeves and corresponding thermocouples (indicated in Figure 1a by $\mathrm{T}_{\mathrm{i}}$, with $\mathrm{i}=1, \ldots, 5$ ), which monitor and control the temperature of the different parts of the oven.

Two Swagelok stainless steel bellow valves are used to allow the evacuation of the oven (evacuation valve) and to separate the Cs reservoir from the experiment vessel (closing valve), respectively. In this way, the Cs reservoir can be refilled with a new ampoule while keeping the experiment in vacuum, and additionally the valve prevents the Cs reservoir from being spoiled if the source is vented.

At ELISE two ovens are installed at the sidewalls and Cs is evaporated into the experiment at a distance of $2.1 \mathrm{~cm}$ from the inner wall through a nozzle of $8 \mathrm{~mm}$ inner diameter. The nozzle of the ovens currently installed at ELISE has an edge cut at $45^{\circ}$ angle, enhancing the evaporation towards the back-plate of the source as shown in Figure 1b. Direct evaporation of Cs towards the plasma grid is in this way avoided, helping to prevent breakdowns in the accelerator.

To monitor the Cs evaporation, a surface ionization detector (oven SID) is located $7 \mathrm{~mm}$ behind the nozzle and is described in details in [12], and here only a brief description is given. The SID consists of two ohmically heated tungsten filaments of $0.3 \mathrm{~mm}$ diameter which are biased against each other by around $50 \mathrm{~V}$. When Cs atoms approach the first tungsten filament (called ionization filament) they are ionized. The $\mathrm{Cs}^{+}$ions are then accelerated and collected onto the second tungsten filament (called ion collector). The resulting SID current flowing between the two filaments is in the range from $\mathrm{nA}$ to $\mathrm{mA}$ (tipically between 2 and $8 \mu \mathrm{A}$ ), and it is proportional to the flux of $\mathrm{Cs}$ atoms impinging on the ionization filament (as long as the current is not limited by space charge effects due to the Child-Langmuir law). Since the oven SID is mounted at the nozzle, the measured current is directly proportional to the evaporation rate of the Cs oven. A calibration of the oven SID can be empirically determined once the $1 \mathrm{~g}$ Cs contained in the oven is completely evaporated: the integral of the entire SID current over time in [Ah] corresponds to the evaporated mass of Cs in [mg], and a calibration factor can be estimated assuming that no Cs is trapped at the oven walls and that the surface conditions on the tungsten filaments do not change. A preliminary calibration of the oven SID results in a typical evaporation rate at the ion source ELISE of around $2 \mathrm{mg} / \mathrm{h}$ per oven, depending on the scenario and isotope. Overall, the ELISE oven ensures stable and reliable Cs evaporations, allowing a wide range of evaporation rates, from below the unity to several tens $\mathrm{mg} / \mathrm{h}$.

\section{Cs oven for SPIDER}

\subsection{Comparison to ELISE oven}

The design of the SPIDER oven is based on the same concept as the ELISE oven: liquid $\mathrm{Cs}$ is evaporated by heating the reservoir. A CAD section drawing of a SPIDER oven is shown in Figure 2a. The main components are highlighted, and the oven is very similar to the one applied at ELISE. The ovens for SPIDER are described in details in [11] and [13], and they are briefly described in the following, focusing on the differences to the ELISE ovens.

The three SPIDER ovens will be installed at the backplate of the ion source. Each oven will besurrounded by $4 \mathrm{RF}$ drivers and will be located in vacuum. As a consenquence, the thermal convection and radiation are negligible and a slow cooling of the reservoir occurs. Moreover, the thermal conduction along the oven has a relevant contribution onto the reservoir temperature: if the temperature of the duct is set to $200^{\circ} \mathrm{C}$, the reservoir temperature cannot reach values below $100^{\circ} \mathrm{C}$. If the duct temperature is set to $250^{\circ} \mathrm{C}$, the reservoir temperature stabilizes around $130^{\circ} \mathrm{C}$. This aspect narrows the choice of temperatures for such oven, defining a minimum 


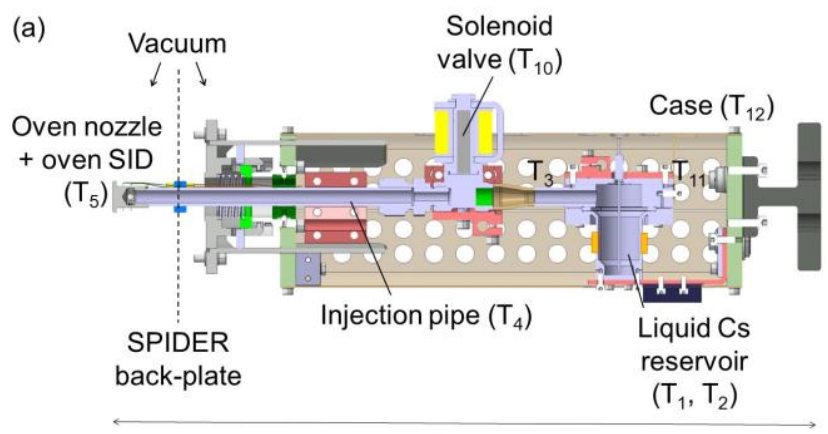

(b)

$\sim 450 \mathrm{~mm}$

Fig. 2. (a) CAD section drawing of the liquid Cs oven for SPIDER. The main components are highlighted and the positions of the 8 thermocouples are indicated by $\mathrm{T}_{\mathrm{i}}$, with $\mathrm{i}=1, . ., 5,10, . ., 12$. (b) Top view of SPIDER ion source with position and orientation of the Cs ovens.

temperature for the reservoir (while the maximum temperature is defined by the oven body temperature, which must be higher than the reservoir temperature). Thus, tests must be performed to characterize the Cs evaporation at different duct and reservoir temperatures.

The oven is mainly made of stainless steel and nickel plated copper. 8 thermocouples are positioned in different spots, as shown in Figure 2a, to monitor the temperature of the oven and the case containing the oven. Only two of these thermocouples control the heating of the oven: $\mathrm{T}_{1}$ controls the temperature of the reservoir which is heated by a band heater surrounding it, while $\mathrm{T}_{4}$ located at the injection tube controls the heating of the oven body, performed by 3 heating cartridges. As a consequence, the temperature of the oven pipe between the reservoir and the valve $\left(\mathrm{T}_{3}\right)$ is usually $60-70^{\circ} \mathrm{C}$ lower than the set temperature of the injection pipe.

The installation in a vacuum environment leads additionallty to the need of remote control. As a consequence, the oven does not contain any ampoule since it can't be externally cracked. The reservoir must be filled with liquid Cs separately from the experiment, and this procedure is performed in a glove box in $\mathrm{Ar}$ atmosphere to avoid any contamination of Cs. To this purpose, the oven was designed to be easily dismantled, and the reservoir can contain few tens of grams of pure Cs to avoid frequent refilling.

The manual closing valve has been replaced by a solenoid valve, which is closed when no current is applied. The valve is not fully metallic, but the sealing is made of KALREZ ${ }^{\circledR} 8900$, a material known for its thermal and chemical stability.

At SPIDER Cs will be evaporated from each oven at a distance of around $1.5 \mathrm{~cm}$ from the back-plate through a $12 \mathrm{~mm}$ diameter nozzle hat, which has 6 equidistant nozzles of $3.5 \mathrm{~mm}$ diameter on the lateral side. In this way, direct evaporation of Cs towards the plasma grid is avoided, and Cs will be probably mostly evaporated towards the back-plate and the sidewalls, as shown in Figure $2 b$. The oven is equipped with an oven SID, whose filaments surround the lateral surface of the nozzle where the orifices are located [11].

\subsection{First tests of the SPIDER oven}

The very first investigations of the SPIDER oven were performed at the dedicated test stand CATS, which is described in detail in [11]. CATS consists of a stainless steel vessel, shown in Figure 3a, separated in two volumes by a stainless steel diaphragm resembling the back-plate of the SPIDER ion source. To study the Cs evaporation several diagnostics are present in the side of the chamber where the nozzle is located (pressure below $10^{-5} \mathrm{mbar}$ ). A tunable diode laser absorption spectroscopy system (TDLAS) serves to measure the line of sight (LOS) integrated Cs density along an absorption length of $48 \mathrm{~cm}$, with an offset between nozzle and LOS axes of $9 \mathrm{~cm}$ [14]. Two SIDs allow determining the Cs outflow from the nozzle (oven SID) and the flux of Cs redistributed in the chamber (movable SID, located close to the walls). For the present preliminary investigations, the two SIDs are not calibrated, but they serve as reference for relative changes of the evaporation from the nozzle.

The tests are devoted to check the system reliability and the stability of the evaporation for different reservoir temperatures, and first results are shown in Figure $3 \mathrm{~b}$. Reservoir temperatures between $100^{\circ} \mathrm{C}$ and $130^{\circ} \mathrm{C}$ are investigated with a duct temperature of $200^{\circ} \mathrm{C}$, while higher reservoir temperatures (between $130^{\circ} \mathrm{C}$ and $160^{\circ} \mathrm{C}$ ) are applied with a duct temperature of $250^{\circ} \mathrm{C}$. For each set of temperatures, the Cs density and SID currents stabilize within ten minutes from the opening of the valve. These signals (and hence the evaporation rate) increase with the reservoir temperature, showing an immediate response to changes of the reservoir temperature. Moreover, signals collected at the same temperature but in different days are very reproducible. Finally, stable signals are observed during the evaporation at constant temperatures over a 4-days campaign (closing the valve during the night, but keeping the heating of the oven on). Thus, the oven ensures a reliable and stable Cs evaporation. 
(a)

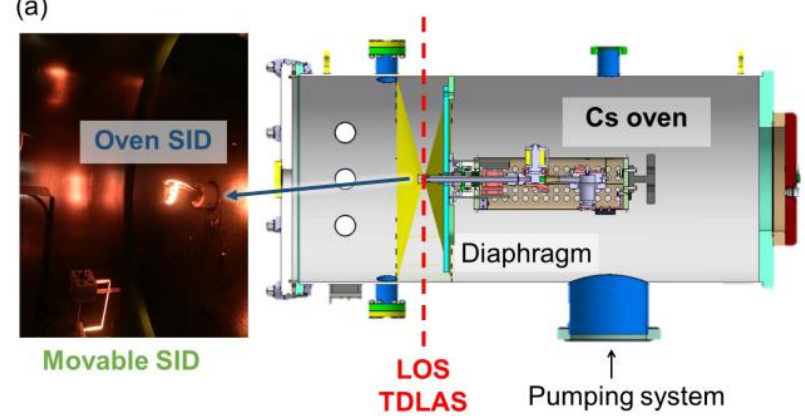

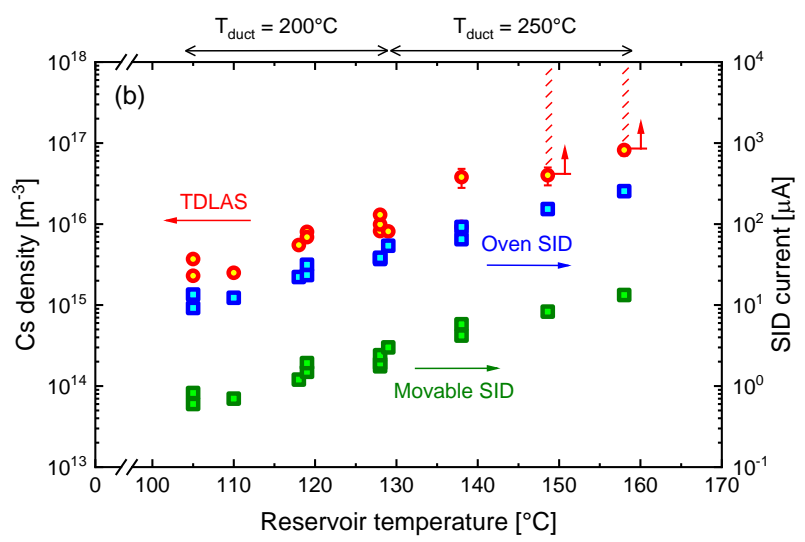

Fig. 3. (a) CAD drawing of the CATS experiment and picture of the left chamber, with highlighted the Cs diagnostics. (b) Cs density measured by TDLAS (in red) and SID currents measured by the oven SID (in blue) and the movable SID (in green) for different reservoir temperatures (with duct temperatures of 200 and $250^{\circ} \mathrm{C}$ ). At temperatures higher than $150^{\circ} \mathrm{C}$, the measured Cs density is a lower limit due to a decreased sensitivity of the system at such high densitites.

\section{Summary}

The Cs ovens applied at ELISE and foreseen at the SPIDER testbed are based on the evaporation of liquid Cs from a reservoir in a Cs oven, and the evaporation of $\mathrm{Cs}$ is controlled by the reservoir temperature.

The ELISE oven is in operation since 2015 and ensures a stable and reliable evaporation, with typical reservoir temperature of $80-130^{\circ} \mathrm{C}$. The ovens allow having evaporation rates from below the unity to several tens $\mathrm{mg} / \mathrm{h}$.

The SPIDER ovens have a different design and nozzle. The ovens are located in vacuum environment, and this leads to a different oven operation with respect to the ELISE oven. One of the newly manufactured SPIDER ovens was successfully tested, showing stable and controllable evaporation of $\mathrm{Cs}$ at applied reservoir temperatures between 100 and $160^{\circ} \mathrm{C}$. As next step, the corresponding evaporation rates will be determined and the reproducibility of the three ovens will be checked.

\section{Acknowledgments}

This work has been carried out within the framework of the EUROfusion Consortium and has received funding from the Euratom research and training programme 20142018 and 2019-2020 under grant agreement number 633053. The views and opinions expressed herein do not necessarily reflect those of the European Commission.

\section{References}

[1] R. Hemsworth, H. Decamps, J. Graceffa and others, "Status of the ITER heating neutral beam system," Nucl. Fusion, vol. 49, p. 045006, 2009.

[2] E. Speth, H. D. Falter, P. Franzen and others, "Overview of the RF source development programme at IPP Garching," Nucl. Fusion, vol. 46, p. S220, 2006.

[3] V. Dudnikov, "Forty years of surface plasma source development," Rev. Sci. Instrum., vol. 83, p. 02A708, 2012.
[4] H. B. Michaelson, "The work function of the elements and its periodicity," J. Appl. Phys., vol. 48, p. 4729, 1977.

[5] R. Gutser, D. Wünderlich, U. Fantz and others, "Dynamics of the transport of ionic and atomic cesium in radio frequency-driven ion sources for ITER neutral beam injection," Plasma Phys. Control. Fusion, vol. 53, p. 105014, 2011.

[6] S. Cristofaro, R. Friedl and U. Fantz, "Simultaneous measurements of work function and $\mathrm{H}^{-}$density including caesiation of a converter surface," AIP Conf. Proc., vol. 1869, p. 030036, 2017.

[7] U. Fantz, C. Hopf, D. Wünderlich and others, "Towards powerful negative ion beams at the test facility ELISE for the ITER and DEMO NBI systems," Nucl. Fusion, vol. 57, p. 116007, 2017.

[8] P. Franzen, B. Heinemann, U. Fantz and others, "Commissioning and first results of the ITERrelevant negative ion beam test facility ELISE," Fusion Eng. Des., vol. 88, p. 3132, 2013.

[9] W. Kraus, D. Wünderlich, U. Fantz and others, "Deuterium results at the negative ion source test facility ELISE," Rev. Sci. Instrum., vol. 89, p. 052102, 2018.

[10] V. Toigo, R. Piovan, S. D. Bello and others, "The PRIMA Test Facility: SPIDER and MITICA testbeds for ITER neutral beam injectors," New J. Phys., vol. 19, p. 085004, 2017.

[11] A. Rizzolo, M. Barbisan, L. Bizzotto and others, "Characterization of the SPIDER Cs oven prototype in the CAesium Test Stand for the ITER HNB negative ion sources," Fusion Eng. Des., vol. 146, p. 676, 2019.

[12] U. Fantz, R. Friedl and M. Fröschle, "Controllable evaporation of cesium from a dispenser oven," Rev. Sci. Instrum., vol. 83, p. 123305, 2012.

[13] A. Rizzolo, M. Pavei and N. Pomaro, "Caesium oven design and R\&D for the SPIDER beam source," Fusion Eng. Des., vol. 88, p. 1007, 2013. 
[14] M. Barbisan, R. Pasqualotto and A. Rizzolo, "Design and preliminary operation of a laser absorption diagnostic for the SPIDER RF source," Fusion Eng. Des., In press, 2019. 
